\title{
High Grade Incidental Brain Arteriovenous Malformation
}

\section{Tarik Zahouani, Sandra Lopez, Leyden Standish-Parkin and Sergey Prokhorov}

Department of Pediatrics of Lincoln Medical and Mental Health Center, Bronx, New York, USA

Corresponding author: Tarik Zahouani, Department of Pediatrics, Lincoln Medical and Mental Health Center, 234E 149th St., Bronx 10451, NY, USA, Tel: +1 718-579-5030; E-mail: Tarikzahouani@gmail.com

Received: 30 November 2016; Accepted: 17 January 2017; Published: 22 January 2017

Citation: Zahouani T, Lopez S, Standish-Parkin L, et al. High Grade Incidental Brain Arteriovenous Malformation. Ann Clin Lab Res. $2017,5: 1$.

\section{Abstract}

Brain arteriovenous malformations (BAVM) are congenital vascular lesions consisting of direct arterial to venous connection without a capillary network. They occur in $0.1 \%$ of the population, most commonly in the supratentorial region. Aneurysm, intracranial hemorrhage and stroke are the major complications. We present 2 cases two cases of high grade incidental BAVM. A thorough evaluation of the lesion and the risks of surgical intervention determine the appropriate management.

Keywords: Brain arteriovenous Malformation, Incidental, Aneurysm, Intracranial hemorrhage, Stroke

\section{Introduction}

Brain arteriovenous malformations (BAVM) are congenital vascular lesions characterized by direct arterial to venous connections without an intervening capillary network. They occur in about $0.1 \%$ of the population and most frequently in the supratentorial region [1]. BAVM are responsible for up to $50 \%$ of spontaneous intracranial hemorrhages and are one of the major causes of stroke in children [2]. The other major medical concern in children with BAVM is the associated aneurysms, which is present in approximately one third of the patients [3]. The management of these lesions is still controversial and is based on a comprehensive evaluation of their natural history as well as the risks of intervention. We present two cases of high grade incidental BAVM.

\section{Case Presentation}

\section{Case 1}

An 18-year old Male with intermittent asthma presented to the emergency department (ED) after sustaining facial, closed head and abdominal injuries following an assault. He had a left sided headache and a brief loss of consciousness but no vision or hearing impairment. On physical examination (PE), the patient appeared well, was alert, oriented and in no acute distress. He had a mild abrasion and hematoma in the left periorbital region and left abdomen with tenderness over the lesions. Heart, lung, abdominal and neurologic examination yielded normal findings. Computed tomography (CT) with angiogram of the brain revealed an AVM in the right occipital lobe, with a nidus measuring $2.4 \mathrm{~cm}$ (Figure 1). No other abnormalities were detected.

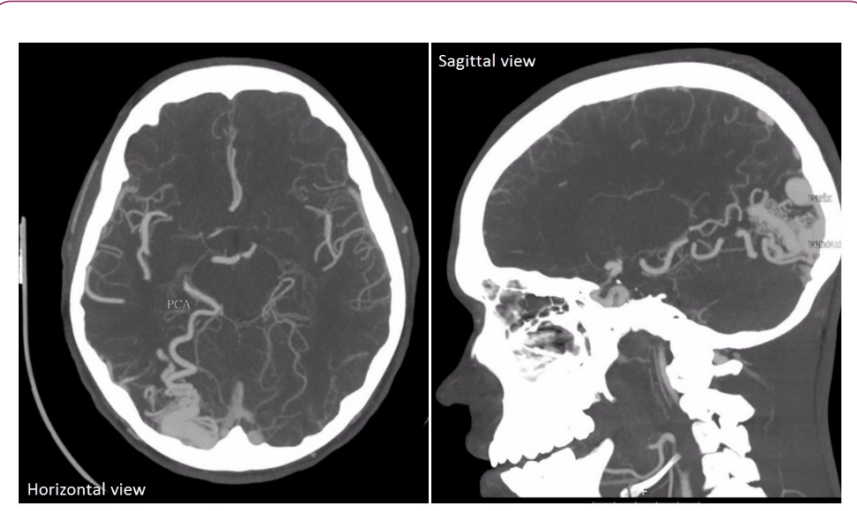

Figure 1 Brain AVM on CT angiogram.

\section{Case 2}

A 17-year old Male with Attention Deficit Hyperactivity Disorder presented to the ED after hitting his head against the door following a fall while on basketball court. He had a left sided headache, dizziness and transient loss of consciousness with no vision or hearing impairment. On PE, the patient was alert, oriented and in no acute distress. He had a $2.5 \mathrm{~cm}$ linear laceration on forehead with no hematomas. Neurologic examination was normal as well as the rest of PE. Brain magnetic resonance imaging (MRI) and magnetic resonance angiogram (MRA) showed $4 \mathrm{~cm} \times 3 \mathrm{~cm}$ AVM in the left inferior parietal lobe extending to the level of the ventricle (Figures 2 and 3).

\section{Discussion}

Brain arteriovenous malformations occur in about $0.1 \%$ of the population and most frequently in the supratentorial region [1]. The most common manifestation of BAVM is an intracranial hemorrhage, accounting for $50 \%$ of initial presentation [4]. The annual hemorrhage rate varies widely from $0.9 \%$ for patients without hemorrhagic AVM presentation, deep AVM location, or deep venous drainage to 
$34.4 \%$ for those harboring all three risk factors [5]. The natural history of high-grade BAVM is incompletely known and hemorrhage rate may vary depending on the methodology used for calculation. Methods that calculate AVM hemorrhage rate from birth to treatment increase the size of the denominator in the person-year method, leading to a low annual hemorrhage rate, whereas methods starting at the time of presentation until treatment decrease the denominator, yielding higher annual rates [2].

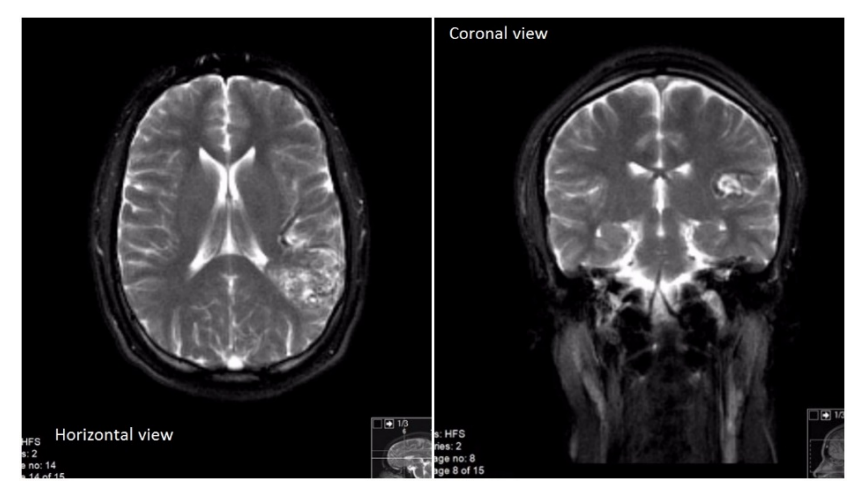

Figure 2 Brain AVM on MRI.

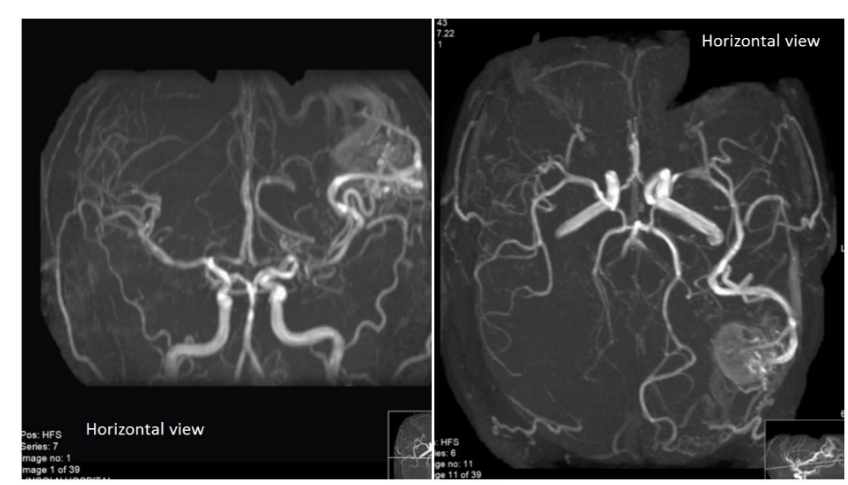

Figure 3 Brain AVM on MRA.

Aneurysms are present in nearly one third of children with BAVM. Anderson et al. studied BAVM associated aneurysms in the pediatric population. They found that there was an association between BAVM associated aneurysms in an arterial location with hemorrhage as 7 of the 10 patients with arterial based aneurysms had radiographic evidence highly suggestive that aneurysm was the source of the hemorrhage. They also found that patients with BAVM associated aneurysms had worse neurological status at discharge, however, there was no significant difference between patients with isolated BAVM and those with BAVM associated aneurysms at long term follow up [3].

The management of BAVM is based on a thorough understanding of the natural history as well as the risks of intervention. Notwithstanding, it is not clear if an intervention improves the outcome of incidentally discovered BAVM. Furthermore, a recent analysis of Columbia database showed that the initiation of any invasive treatment strategy was associated with a threefold increased risk of BAVM hemorrhage as well as an increased risk of clinical impairment [6].

Even though the management of unruptured BAVMs has come under significant scrutiny as a result of the randomized trial of unruptured brain arteriovenous malformations (ARUBA), the observations in that study have little relevance to pediatric patients, given that children were excluded from the ARUBA study, pediatric patients are anatomically and physiologically different from adults, and that the length of its clinical follow up was shorter (the mean length of follow up was less than 3 years). Nonetheless, a review of the ARUBA study is relevant, as its data reveal an annual stroke rate of approximately $4 \%$ for untreated AVMs [7].

\section{Conclusion}

Brain arteriovenous malformations discovered incidentally are more frequent due to the availability of high quality noninvasive imaging studies. Hemorrhage is a major complication of these lesions, especially when they are associated with aneurysms that are arterially located. The management of BAVM is still controversial and should be based on a thorough understanding of the natural history as well as the risks of intervention.

\section{References}

1. Mohr JP, Kejda-Scharler J, Pile-Spellman J (2013) Diagnosis and treatment of arteriovenous malformations. Curr Neurol Neurosci Rep 13: 324.

2. Darsaut TE, Guzman R, Marcellus ML (2011) Management of pediatric intracranial arteriovenous malformations: Experience with multimodality therapy. Neurosurgery 69: 540-556.

3. Anderson RC, McDowell MM, Kellner CP (2012) Arteriovenous malformation-associated aneurysms in the pediatric population. J Neurosurg Pediatr 9: 11-16.

4. Chalouhi N, Dumont AS, Randazzo C (2011) Management of incidentally discovered intracranial vascular abnormalities. Neurosurg Focus 31: e1.

5. Stapf C, Mast H, Sciacca RR (2006) Predictors of hemorrhage in patients with untreated brain arteriovenous malformation. Neurology 66: 1350-1355.

6. Stapf C, Mohr JP (2007) Unruptured brain arteriovenous malformations should be treated conservatively: Yes. Stroke 38: 3308-3309.

7. Gross BA, Storey A, Orbach DB (2015) Microsurgical treatment of arteriovenous malformations in pediatric patients: The Boston children's hospital experience. J Neurosurg Pediatr 15: 71-77. 